\title{
Mobile epifauna on subtidal brown seaweeds in northeastern New Zealand
}

\author{
Richard B. Taylor, Russell G. Cole* \\ Leigh Marine Laboratory and School of Biological Sciences, University of Auckland, PO Box 349, Warkworth, New Zealand
}

\begin{abstract}
This paper examines the distribution and abundance of mobile epifauna $>1 \mathrm{~mm}$ inhabiting 10 species of subtidal brown seaweeds (Phaeophyceae) in northeastern New Zealand. Cammarid amphipods and isopods were the most abundant animals captured, while a diverse group of gastropods was also present at lower densities. Finely structured seaweeds such as Carpophyllum plumosum var. capillifolium and Cystophora retroflexa tended to support far more animals (up to $2000 \mathrm{ind}$. per $100 \mathrm{~g}$ algal wet wt) than did coarsely structured seaweeds. Comparison of epifaunas among C. plumosum growth forms of varying thallus width indicated that this pattern was due to the morphology of the plants rather than differences in their internal composition. There was a trend for isopods with tubular body shapes to live on algal species with narrow fronds, and for dorso-ventrally flattened isopods to live on algae with wide fronds. Most of the seaweed species held epifaunal assemblages that were distinct from one another in multivariate space, but the individual epifaunal taxa were generally not strongly host-plant specific, with most occurring on more than 1 algal species. It is suggested that most of the epifauna have a weak relationship with their host plant. Epifaunal densities on Ecklonia radiata peaked at $6 \mathrm{~m}$ depth, and declined with increasing depth.
\end{abstract}

KEY WORDS: New Zealand Seaweed Algae Epifauna Habitat structure Amphipod Isopod Gastropod

\section{INTRODUCTION}

The large brown seaweeds (Phaeophyceae) of shallow temperate reefs harbour numerous small mobile animals (Edgar \& Moore 1986). Small crustaceans (amphipods, isopods, and copepods) and gastropods are frequently abundant, and may be an important food source for juvenile fishes which are also abundant in macroalgal stands (Bray \& Ebeling 1975, Jones 1988). The invertebrates are taxonomically and morphologically diverse, and exhibit a range of trophic habits. They may filter feed (Caine 1977), graze epiphytic algae (Brawley \& Fei 1987), eat detritus (Zimmerman et al. 1979), prey upon other epifauna (Roland 1978), or consume the host plant itself (Duffy 1990). The grazing activity of the latter group may become sufficiently intense to remove large macroalgae (Tegner \& Dayton 1987). However, compared to the well

\footnotetext{
- Present address: Department of Earth Sciences, University of Waikato, Private Bag 3105, Hamilton, New Zealand
}

known epifaunas of seagrasses (e.g. Nelson 1979, Stoner 1980), information on the ecology of the mobile epifauna of large brown algae is sparse. This is especially true for New Zealand, where published information is limited to the popular treatment of seaweed fauna by Morton \& Miller (1968), studies of Jansen (1971) on intertidal sphaeromatid isopods, Hicks (1977a, b) on phytal harpacticoid copepods, and Kingsford \& Choat (1985) on epifaunas of attached and drift seaweed described at coarse taxonomic levels. Here we report the results of a survey of the epifauna on subtidal large brown algae in northeastern New Zealand.

Subtidal rocky reefs on exposed coasts in mainland northeastern New Zealand are inhabited by fucalean and laminarian seaweeds which show predictable patterns of abundance with depth (Choat \& Schiel 1982, Schiel 1988). Members of the fucalean genera Carpophyllum, Cystophora, Landsburgia, Sargassum, and Xiphophora, and the laminarians Ecklonia radiata and Lessonia variegata typically occur in mixed stands to 
about $5 \mathrm{~m}$ below mean low water spring. Macroalgae are often absent between depths of 5 and $10 \mathrm{~m}$, with this zone dominated by the sea urchin Evechinus chloroticus and a characteristic guild of grazing gastropods (Choat \& Andrew 1986). However, stands of Carpophyllum flexuosum sometimes occur in these areas (R. Cole unpubl. data). Below $10 \mathrm{~m}$ monospecific stands of Ecklonia radiata dominate. In sheltered localities the urchin-grazed zone is frequently narrower, and hard substrata below $5 \mathrm{~m}$ may be dominated by monospecific stands of $C$. flexuosum, or mixed stands of E. radiata and C. flexuosum (Grace 1983). Most individual seaweed plants are free of conspicuous algal epiphytes (authors' pers obs.). Illustrations of the seaweeds can be found in Lindauer et al. (1961) and Morton \& Miller (1968).

Here we describe the epifaunas of these brown algal species at a number of sites, and for Ecklonia radiata at a number of depths within sites. Epifaunal abundances and species composition can be strongly influenced by host plant morphology (e.g. Edgar 1983a, Hacker \& Steneck 1990, Holmlund et al. 1990). We relate epifaunal abundances to algal thallus width, and attempt to separate effects of algal morphology from internal plant composition by examining the epifaunas of several growth forms of 1 algal species, Carpophyllum plumosum. For 1 epifaunal group, the isopods, body shape is investigated as a factor potentially influencing distribution among host algal species.

\section{METHODS}

Specimens of the 10 most abundant large brown algal species were sampled at 17 sites near Leigh $\left(36^{\circ} 17^{\prime} \mathrm{S}, 174^{\circ} 48^{\prime} \mathrm{E}\right)$ on the northeastern coast of New Zealand (Fig. 1). Sampling was carried out in 1991, 1992 and 1993 from June to September (austral winter and early spring), when sea surface temperatures ranged from 12.5 to $15^{\circ} \mathrm{C}$. Sea surface temperatures at Leigh typically range from 14 to $21^{\circ} \mathrm{C}$ annually (Evans 1992).

Plants for sampling were haphazardly selected underwater, gently enclosed in a large plastic bag, and cut off $1 \mathrm{~cm}$ above the holdfast. The mouth of the bag was sealed with a cord noose. Unless otherwise stated, 5 replicate plants were collected. At the laboratory each plant was washed vigorously in a bucket containing $5 \mathrm{l}$ freshwater with $10 \mathrm{ml}$ formalin added to irritate the epifauna and cause them to release their grip on the plant. Epifauna remaining in the bucket and sampling bag were washed onto a $1 \mathrm{~mm}$ mesh sieve. This procedure was carried out twice for each plant. The process removes over $99 \%$ of individuals of the abundant amphipod Podocerus manawatu from Carpophyl- lum plumosum var capillifolium (R. Taylor unpubl. data). The plant was then weighed $( \pm 1 \mathrm{~g})$ after shaking off excess water All animals retained on the sieve were counted, and their densities expressed as numbers per $100 \mathrm{~g}$ of algal wet weight. Texts used to identify the animals were Richardson \& Yaldwyn (1958), Hurley (1961), McCain (1969), Barnard (1972), Melrose (1975), Riek (1976), Wilson et al. (1976), Hurley \& Jansen (1977), Powell (1979), Lowry (1981), Poore (1981), McLay (1988), Hardy (1989), Paulin et al. (1989), Barnard \& Karaman (1991), and Poore \& Lew Ton (1993). A reference collection of specimens has been lodged with the Auckland Institute and Museum, Auckland, New Zealand (AK 83657-83803).

Interspecific variation. Ten species of algae were collected at similar depths from 16 sites as they were encountered (see Table 1).

Canonical discriminant analysis (CDA) was used to examine the uniqueness of the epifaunal assemblages on the different algal species from the 16 sites, and to identify the epifaunal taxa which best discriminated among the algal species. CDA is an ordination technique (SAS Institute Inc. 1987), which we used to reduce multidimensional data (consisting of densities of many epifaunal taxa) to 2 dimensions, which were displayed graphically. The 25 most common epifaunal taxa (densities standardised to mean per $100 \mathrm{~g}$ algae for each algal species) were selected for this analysis (see Table 2). Taxa that had consistently highly positive or consistently highly negative values for all of the total-, between-, and pooled within-canonical structures of the analysis were considered to be those showing largest density differences among the seaweed species, and were labelled on the appropriate axes.

Effects of algal morphology. Algal thallus width was measured to enable a crude ranking of algal species and growth forms (listed below) in terms of their morphological complexities. Plants were laid out flat $(n=8$ individuals per species/growth form), and their thallus width measured at 10 haphazardly chosen points (the holdfast was removed beforehand). We did not subdivide the algae into stipe, fronds, etc., because we had no knowledge of within-plant epifaunal distribution and so had to assume they were utilising the whole plant. A grand mean was calculated for each algal species/growth form. The common isopod taxa were divided into 2 body shape types, and their relative abundances related to the mean thallus width of their host plants. Arcturidae, Batedotea elongata, and Paranthura spp. had vermiform body shapes ('tubular'), and Amphoroidea spp., Plakarthrium typicum, and miscellaneous Sphaeromatidae were dorsoventrally flattened ('flat').

In an attempt to separate the effects of algal morphology from internal plant composition, we examined 
a) Northern New Zealand

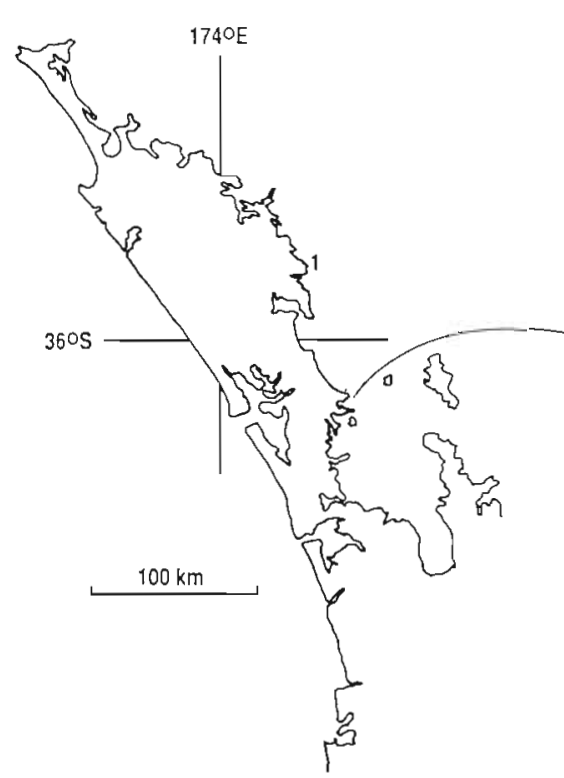

b) Leigh

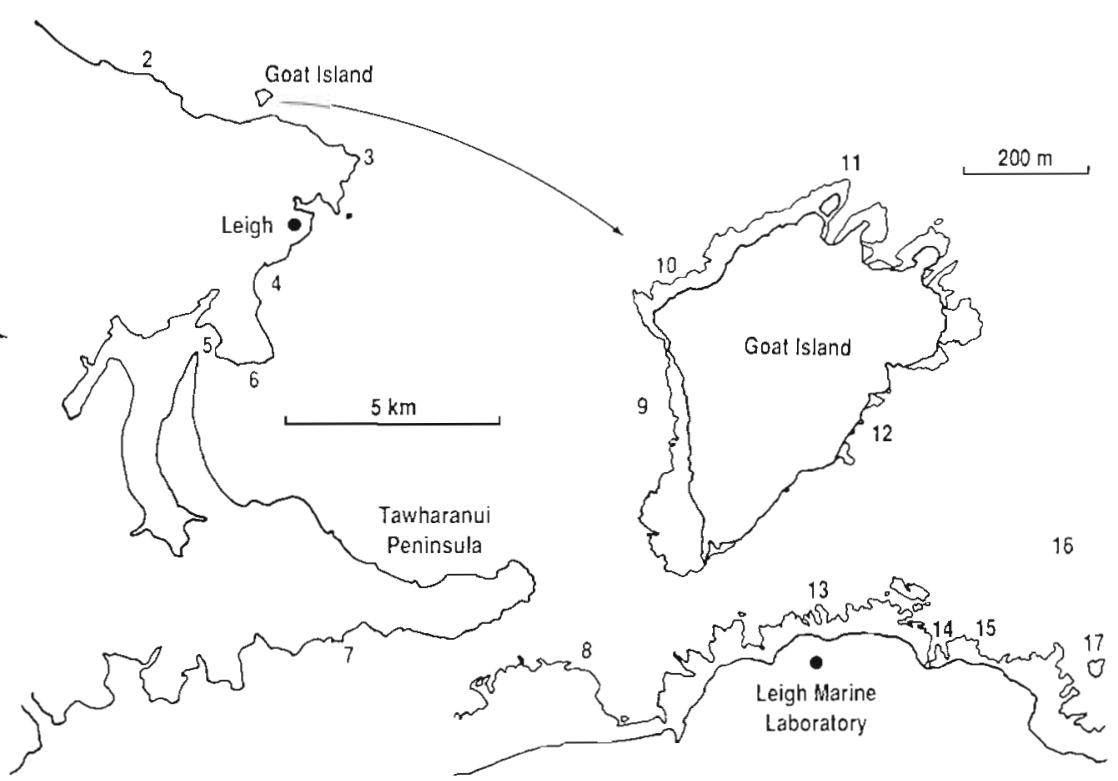

Fig. I Sampling sites: (1) Tutukaka, (2) Okakari Point, (3) Cape Rodney, (4) Matheson Bay, (5) Ti Point Wharf, (6) Ti Point, (7) Matatuahu Point, (8) Echinoderm Reef, (9) Alphabet Bay, (10) Schiels Pool, (11) Splendid Reef, (12) Goat Island Channel, (13) Pumphouse Reef, (14) Waterfall Gutter, (15) Inner Waterfall Reef, (16) Waterfall Crest, (17) Ray Rock

the epifaunas of several growth forms of 1 algal species, Carpophyllum plumosum. Two forms are described by Lindauer et al. (1961). The most common near Leigh is the finely dissected $C$. plumosum var. capillifolium, which typically has a single flattened stipe of approximately $5 \mathrm{~mm}$ width and $1 \mathrm{~m}$ length, with dense clumps of frondlets growing in a continuous zone along the margins, so as to give the appearance of a bottle brush (hereafter 'typical/fronded'). $C$. plumosum is less common and has much wider, flattened fronds. Furthermore, at some shallow-water exposed sites there is a form of C. plumosum var. capillifolium which consists mainly of stipe, having sparse short frondlets, the bases of which often support small clumps of epiphytic red algae (mostly Antithamnion applicitum and Ceramium spp.) (hereafter 'frondless/epiphytized'). Specimens of these growth forms were sampled at Waterfall Crest and Okakari Point, where they grew within meters of each other (see Table 3). A reciprocal transplant experiment was conducted to see whether differences in epifaunal density and composition were due to differences between plant growth forms or to small-scale spatial variation in some other factor. In August 1991 at Okakari Point, 5 typical/fronded C. plumosum var. capillifolium, and 5 frondless/epiphytized C. plumosum var. capillifolium were gently detached from the substratum, denuded of epifauna by shaking at the surface (this removed $97.4 \%$ of the epifauna; R. Taylor unpubl. data), and transplanted into nearby beds of the other growth form by wiring their holdfasts to masonry nails hammered into the bedrock. The plants were collected and processed 10 d later as described above.

Depth-related variation of epifauna on Ecklonia radiata. Depth-related changes in epifaunal densities were examined by collecting Ecklonia radiata plants $(\mathrm{n}=3$ ) at $3 \mathrm{~m}$ depth intervals from the intertidal to the bottom of the reef at Splendid Reef and Ray Rock (Fig. 1). The other algal species were not sampled as they had much narrower depth ranges (Choat \& Schiel 1982, Schiel 1988).

\section{RESULTS}

\section{Interspecific variation in epifaunal composition}

Carpophyllum plumosum var. capillifolium and Cystophora retroflexa clearly supported far higher epifaunal densities than did the other seaweed species surveyed, with means typically ranging from 500 to 2000 animals per $100 \mathrm{~g}$ algal weight, compared with 10 to 350 for the rest (Table 1). The 2 laminarians, Ecklonia radiata and Lessonia variegata, held the lowest epifaunal densities. However, there was considerable spatial variation in epifaunal densities within seaweed species. For example, the mean epifaunal density on $E$. radiata at Waterfall Crest was higher than those on $C$. 
Table 1. Mean densities of epifauna per $100 \mathrm{~g}$ algae $(\mathrm{n}=5)$. Standard errors averaged $22.8 \%$ of the mean. Algal species are ranked in declinung order of grand mean total epifaunal density. $\mathrm{Cr}=$ Cystophora retroflexa, $\mathrm{Cpc}=$ Carpophyllum plumosum var. capillifolium, $\mathrm{Ct}=$ Cystophora torulosa, $\mathrm{Xc}=$ Xiphophora chondrophylla, $\mathrm{Cf}=$ Carpophyllum flexuosum, Lq $=$ Landsburgia quercifolia, $\mathrm{Cm}=$ Carpophyllum maschalocarpum, $\mathrm{Ss}=$ Sargassum sinclairi, $\mathrm{Er}=$ Ecklonia radiata, Lv = Lessonia variegata

\begin{tabular}{|c|c|c|c|c|c|c|c|c|c|c|}
\hline \multirow[t]{2}{*}{ Site } & \multicolumn{10}{|c|}{ Algal species } \\
\hline & $\mathrm{Cr}$ & Cpc & $\mathrm{Ct}$ & $\mathrm{Xc}$ & $\widetilde{C f}$ & $\mathrm{Lq}$ & $\mathrm{Cm}$ & Ss & Er & LV \\
\hline Tutukaka & & & & & & & & & & 13 \\
\hline Okakari Point & & 1466 & & & 90 & & 25 & & 52 & \\
\hline Echinoderm Reef & 1180 & 1951 & 356 & 96 & & & & & & \\
\hline Alphabet Bay & & & & & & & & 86 & 8 & \\
\hline Schiels Pool & & 606 & & & 250 & & 90 & & 138 & \\
\hline Splendid Reef & & & & & 50 & 31 & & & & \\
\hline Goat Island Channel & & & & & 150 & & & & 24 & \\
\hline Pumphouse Reef & & 122 & & & & & & & & \\
\hline Waterfall Gutter & & 168 & & & & & & & & \\
\hline Waterfall Crest & & 273 & & & 75 & & 42 & & 149 & \\
\hline Inner Waterfall Reef & & & & & & 43 & & & & \\
\hline Cape Rodney & & & & 348 & & 182 & & 51 & & \\
\hline Matheson Bay & 567 & & 307 & & & & & & & \\
\hline Ti Point & & 556 & & & 105 & & 170 & & 39 & \\
\hline Ti Point Wharf & & & & & 17 & & & & & \\
\hline Matatuahu Point & & & & & 76 & & & & 14 & \\
\hline Grand mean & 874 & 735 & 332 & 222 & 102 & 85 & 82 & 69 & 61 & 13 \\
\hline
\end{tabular}

plumosum var. capillifolium at Pumphouse Reef and Waterfall Gutter.

Seventy-three taxa of mobile epifauna were found (Table 2). Gammarid amphipods and isopods were particularly important, contributing 18 of the 20 most abundant taxa. Gastropods were represented by many species, although their individuals were far less numerically abundant, Overall, the epifaunal taxa were not highly host-plant specific. Seven taxa (9.6\%) were recorded from all 10 algal species, and only 20

Table 2. Mobile epifauna found on 10 species of seaweed. Numbers are mean densities per $100 \mathrm{~g}$ algae calculated across all individual plants collected. N: number of algal species that epifaunal taxon is found on. Epifaunal taxa are ranked in declining order of average mean density across all seaweed species surveyed. For seaweed species codes see Table 1. For epifaunal taxonomic authorities see references cited in methods. $\mathrm{B}=$ brachyuran, $\mathrm{C}=$ caprellid amphipod, $\mathrm{CS}=$ caridean shrimp, $\mathrm{E}=\mathrm{echinoderm}$, $\mathrm{F}=$ fish, $\mathrm{G}=$ gammarid amphipod, $\mathrm{GS}=$ gastropod, $\mathrm{I}=$ isopod, $\mathrm{M}=$ mysid shrimp, $\mathrm{P}=$ platyhelminth, $\mathrm{T}=$ trichopteran (insect)

\begin{tabular}{|c|c|c|c|c|c|c|c|c|c|c|c|c|}
\hline \multirow[t]{2}{*}{ Epifaunal taxon } & \multicolumn{10}{|c|}{ Algal species } & \multirow[t]{2}{*}{ Mean } & \multirow[t]{2}{*}{$\mathrm{N}$} \\
\hline & $\mathrm{Cf}$ & $\mathrm{Cm}$ & $\mathrm{Cpc}$ & Cr & $\mathrm{Ct}$ & Er & Lq & Lv & Ss & $\mathrm{Xc}$ & & \\
\hline Podocerus manawatu (G) & 10.17 & 12.74 & 437.61 & 708.10 & 132.87 & 0.21 & 0.07 & & 2.38 & 8.06 & 131.22 & 9 \\
\hline Ischyroceridae $(G)$ & 23.48 & 22.42 & 83.59 & 14.56 & 48.16 & 24.07 & 6.67 & 8.49 & 7.40 & 89.76 & 32.86 & 10 \\
\hline Podocerus wanganui (G) & 0.60 & 0.43 & 11.43 & 7.43 & 3.69 & 0.02 & 47.40 & 1.35 & 4.55 & 77.06 & 15.40 & 10 \\
\hline Hyale spp. (G) & 1.11 & 3.30 & 42.45 & 6.83 & 47.13 & 0.25 & 2.91 & 0.04 & 0.29 & 5.59 & 10.99 & 10 \\
\hline Podocerus karu (G) & 2.67 & 3.67 & 44.77 & 8.51 & 21.07 & 0.14 & 0.08 & 0.03 & 5.74 & 4.25 & 9.09 & 10 \\
\hline Stenothoe spp. (G) & 0.15 & 0.42 & 11.00 & 44.55 & 21.38 & 0.08 & 0.51 & 0.79 & 0.19 & 5.19 & 8.43 & 10 \\
\hline Eatoniella spp. (GS) & 2.23 & 1.08 & 31.69 & 40.59 & 2.71 & 1.44 & & & 0.28 & 1.43 & 8.15 & 8 \\
\hline Misc. Sphaeromatidae ${ }^{b}$ (I) & 3.65 & 2.26 & 3.22 & 14.44 & 8.36 & 0.06 & 8.98 & & 2.02 & 6.27 & 4.93 & 9 \\
\hline Plakarthrium typicum (I) & 3.35 & 3.45 & 0.38 & 0.26 & 0.03 & 21.96 & 10.10 & 0.97 & 6.87 & 0.41 & 4.78 & 10 \\
\hline Ampithoe spp. (G) & 20.12 & 10.12 & 12.57 & & 0.21 & 0.08 & 3.21 & & 0.40 & & 4.67 & 7 \\
\hline Arcturidae (I) & 7.88 & 4.05 & 4.05 & 10.17 & 2.74 & 0.09 & 0.13 & 0.12 & 5.22 & 6.14 & 4.06 & 10 \\
\hline Aora maculata (G) & 9.26 & 4.41 & 12.90 & 0.84 & 0.13 & 4.94 & 0.04 & & 5.65 & 0.51 & 3.87 & 9 \\
\hline Batedotea elongata (I) & 1.27 & 0.53 & 8.94 & 8.55 & 4.58 & 0.02 & 0.45 & & 1.03 & 3.16 & 2.85 & 9 \\
\hline Gammaropsis spp. (G) & & 0.27 & 4.70 & 1.56 & 15.87 & & 0.03 & & & 0.12 & 2.25 & 6 \\
\hline Parapherusa crassipes (G) & & & & & 17.06 & & & & & & 1.71 & 1 \\
\hline Misc. Eusiridae (G) & & & 0.29 & 1.83 & 1.85 & 0.02 & 0.02 & & 5.50 & 3.85 & 1.33 & 7 \\
\hline Amphoroidea media (I) & 5.50 & 4.46 & 1.40 & 0.05 & & 0.01 & 0.40 & & 0.19 & 0.12 & 1.21 & 8 \\
\hline Hippolyte bifidirostris (CS) & 1.05 & 0.69 & 1.80 & 1.51 & 0.07 & 0.33 & & & 4.98 & & 1.04 & 7 \\
\hline Gondogeneia spp (G) & & & 0.18 & & 0.33 & & 0.56 & & 2.24 & 6.56 & 0.99 & 5 \\
\hline Ampithoe lessoniae (G) & 3.94 & 2.88 & 0.77 & & & & & & & & 0.76 & 3 \\
\hline
\end{tabular}


Table 2 (continued)

\begin{tabular}{|c|c|c|c|c|c|c|c|c|c|c|c|c|}
\hline \multirow{2}{*}{ Epifaunal taxon } & \multicolumn{10}{|c|}{ Algal species } & \multirow[t]{2}{*}{ Mean } & \multirow[t]{2}{*}{$N$} \\
\hline & $\mathrm{Cf}$ & $\mathrm{Cm}$ & Cpc & $\mathrm{Cr}$ & $\mathrm{Ct}$ & Er & $\mathrm{Lq}$ & Lv & Ss & $\mathrm{Xc}$ & & \\
\hline Caprellina longicollis (C) & 0.17 & 0.02 & 4.24 & 0.34 & & 0.05 & 0.24 & 0.03 & 2.37 & 0.07 & 0.75 & 9 \\
\hline \multicolumn{13}{|l|}{ Micrelenchus sanguineus } \\
\hline sanguineus (GS) & 0.45 & 0.20 & 2.08 & 1.10 & 0.71 & 0.30 & 0.05 & 0.03 & 2.16 & & 0.71 & 9 \\
\hline Amphoroidea longipes (I) & 0.01 & 0.03 & & & & 3.19 & 2.72 & 0.59 & 0.19 & 0.14 & 0.69 & 7 \\
\hline Tetradeion crassum (G) & 0.19 & 0.16 & 2.90 & 0.08 & 0.39 & 0.14 & 0.06 & & 0.17 & 0.50 & 0.46 & 9 \\
\hline Atyloella spp (G) & 0.39 & 0.99 & 1.35 & & & 0.63 & & & 1.13 & & 0.45 & 5 \\
\hline Siriella denticulata (M) & 0.23 & 0.35 & & 0.08 & & 0.40 & & 0.06 & 2.25 & & 0.34 & 6 \\
\hline Paguroidea & 0.44 & 0.42 & 0.59 & & 0.08 & 0.04 & & & 1.69 & & 0.33 & 6 \\
\hline Paradexamine spp. (G) & 0.20 & 0.01 & 2.93 & & & & & & & & 0.31 & 3 \\
\hline Misc. Polychaeta & 0.55 & 0.08 & 1.19 & 0.19 & 0.48 & 0.01 & & & 0.50 & 0.12 & 0.31 & 8 \\
\hline Tanaidacea & 0.45 & 0.03 & 0.47 & 0.38 & 0.47 & & & 0.03 & 0.45 & & 0.23 & 7 \\
\hline Ceinidae $\{\mathrm{G}\}$ & 0.11 & 0.58 & 0.23 & & & 0.87 & & & & 0.11 & 0.19 & 5 \\
\hline Neogastropoda (GS) & 0.08 & 0.30 & 0.24 & 0.32 & 0.06 & & 0.11 & & 0.37 & 0.12 & 0.16 & 8 \\
\hline Cantharidus purpureus (GS) & 0.16 & 0.49 & 0.10 & & & 0.55 & 0.07 & 0.06 & & & 0.14 & 6 \\
\hline Incisura lytteltonensis (GS) & & & 0.09 & 0.25 & 0.20 & & & & & 0.79 & 0.13 & 4 \\
\hline Micrelenchus dilatatus (GS) & 0.01 & 0.23 & 0.07 & 0.42 & 0.29 & & 0.07 & & & 0.15 & 0.12 & 7 \\
\hline Cookia sulcata (GS) & 0.24 & 0.14 & 0.26 & & & 0.02 & & 0.04 & 0.22 & & 0.09 & 6 \\
\hline Caprella equilibra (C) & 0.05 & & 0.34 & & & & & 0.14 & 0.38 & & 0.09 & 4 \\
\hline Rhynchocoela & 0.01 & 0.05 & 0.77 & & & & & & & & 0.08 & 3 \\
\hline Eatoniella (Pellax) huttoni (GS) & 0.15 & 0.05 & 0.53 & & & 0.01 & & & & & 0.07 & 4 \\
\hline \multicolumn{13}{|l|}{ Eatoniella (Dardanula) } \\
\hline limbata (GS) & 0.22 & & 0.26 & & & & & & 0.20 & & 0.07 & 3 \\
\hline Elasmopus neglectus (G) & & & & & & & & & & 0.54 & 0.05 & 1 \\
\hline Paranthura flagellata (I) & & 0.11 & 0.02 & 0.26 & 0.10 & & & & & & 0.05 & 4 \\
\hline Stylochoplana spp. (P) & 0.24 & 0.03 & 0.18 & & & 0.04 & & & & & 0.05 & 4 \\
\hline Gitanopsis squamosa (G) & & & 0.08 & 0.06 & 0.10 & & & & 0.25 & & 0.05 & 4 \\
\hline Philanisus plebeius $(\mathrm{T})$ & 0.05 & & 0.42 & & & & & & & & 0.05 & 2 \\
\hline Trochus (Thorista) viridis (GS) & 0.14 & 0.10 & & & & 0.18 & & & & & 0.05 & 3 \\
\hline Halicarcinus innominatus (B) & & & 0.34 & & & 0.01 & & & & & 0.04 & 2 \\
\hline Gastrocyathus gracilis $(\mathrm{F})$ & 0.03 & & & & & 0.06 & 0.25 & & & & 0.03 & 3 \\
\hline \multicolumn{13}{|l|}{ Limnoria (Phycolimnoria) } \\
\hline stephenseni (I) & 0.06 & & & & & & 0.07 & & 0.19 & & 0.03 & 3 \\
\hline Notoclinus compressus $(\mathrm{F})$ & 0.05 & & 0.12 & & 0.05 & & 0.04 & & & & 0.03 & 4 \\
\hline Thoristella oppressa (GS) & & & & & 0.25 & & & & & & 0.03 & 1 \\
\hline Pycnogonida & & & 0.23 & & & 0.01 & & & & & 0.02 & 2 \\
\hline Maoricrypta costata (GS) & 0.11 & & & & & 0.12 & & & & & 0.02 & 2 \\
\hline Parawaldeckia spp. (G) & & & 0.17 & & & & & & & & 0.02 & 1 \\
\hline Runnica katipoides (GS) & & 0.01 & 0.10 & 0.04 & & & & & & & 0.02 & 3 \\
\hline Tricladida $(\mathrm{P})$ & & & & & & 0.09 & & & & & 0.01 & 1 \\
\hline Lamellaria ophione (GS) & & & 0.05 & & 0.03 & & & & & & 0.01 & 2 \\
\hline Maurea punctulata (GS) & 0.02 & & & & & & & 0.06 & & & 0.01 & 2 \\
\hline Acmaeidae (GS) & & & 0.07 & & & & & & & & 0.01 & 1 \\
\hline Cerapus harfootus (G) & & & 0.07 & & & & & & & & 0.01 & 1 \\
\hline Paranthura sp. (I) & & & & 0.05 & & & & & & & 0.01 & 1 \\
\hline Forsterygion lapillum $(F)$ & & 0.05 & & & & & & & & & 0.005 & 1 \\
\hline Notomithrax spp. (B) & & & 0.04 & & & & & & & & 0.004 & 1 \\
\hline \multicolumn{13}{|l|}{ Maoricrypta (Zeacrypta) } \\
\hline monoxyla (GS) & & & 0.04 & & & & & & & & 0.004 & 1 \\
\hline Bivalvia & & & & 0.04 & & & & & & & 0.004 & 1 \\
\hline Doto pita (GS) & 0.03 & & & & & & & & & & 0.003 & 1 \\
\hline Polyplacophora & 0.02 & & & & & & & & & & 0.002 & 1 \\
\hline Coscinasterias calamaria (E) & & & 0.02 & & & & & & & & 0.002 & 1 \\
\hline Turbo smaragdus (GS) & 0.01 & & & & & & & & & & 0.001 & 1 \\
\hline Cantharidus opalus opalus (GS) & & 0.01 & & & & & & & & & 0.001 & 1 \\
\hline Corophium acutum (G) & 0.01 & & & & & & & & & & 0.001 & 1 \\
\hline Eubranchus agrius (GS) & 0.01 & & & & & & & & & & 0.001 & 1 \\
\hline Evechinus chloroticus (E) & 0.01 & & & & & & & & & & 0.001 & 1 \\
\hline Total taxa & 49 & 40 & 52 & 30 & 31 & 35 & 26 & 16 & 32 & 25 & & \\
\hline Total individuals sampled & 5861 & 4178 & 20489 & 16959 & 6121 & 5234 & 2975 & 302 & 387 & 1348 & & \\
\hline
\end{tabular}


taxa (27.4\%) were found on just 1 algal species. This latter group was mostly represented by relatively few individuals, so their apparent specificity is more likely due to their overall rarity than to strong habitat preferences.

The CDA run on densities of the 25 commonest taxa showed clearly that most of the seaweed species had distinct epifaunas, especially Cystophora retroflexa and Carpophyllum plumosum var. capillifolium (Fig. 2). The gammarid amphipod genus Stenothoe was important for distinguishing the epifauna of $C$. retroflexa and to a lesser extent $C$. plumosum var. capillifolium along canonical variate 1 (CV 1), while the sphaeromatid isopod Amphoroidea longipes characterised Ecklonia radiata. Presence of the gammarid amphipod genus Ampithoe and absence of the isopod Plakarthrium typicum identified the epifauna of $C$. plumosum var. capillifolium and to a lesser extent $C$. flexuosum.

Several epifaunal taxa featured prominently across the 10 algal species surveyed (Fig. 3). The gammarid amphipod family Ischyroceridae (IS on the figure) was the most consistently common epifaunal taxon, being the most abundant taxon on 6 of the 10 algal species, and not ranked lower than fourth on any algal species. The gammarid Podocerus manawatu (PM) was the most abundant taxon on 3 algal species, reaching very high mean densities on Carpophyllum plumosum var. capillifolium and Cystophora retroflexa, and comprising the majority of the epifaunal community on these seaweeds. Other common taxa were the gammarids Podocerus karu (PK) and Aora maculata (AO).

\section{Effects of algal morphology}

In order of increasing mean thallus width, the algal species were: Carpophyllum plumosum var. capillifolium (mean $=1.55 \mathrm{~mm}, \mathrm{SE}=0.17, \mathrm{n}=8$ plants with 10 points measured on each), Cystophora retroflexa $(1.56 \pm 0.14)$, Xiphophora chondrophylla (1.94 \pm 0.09), Cystophora torulosa (2.21 \pm 0.12$)$, Carpophyllum maschalocarpum (4.71 \pm 0.28), Landsburgia quercifolia $(6.03 \pm 0.26)$, Carpophyllum flexuosum $(6.64 \pm 0.59)$, Sargassum sinclairii (10.56 \pm 0.83), Lessonia variegata $(28.03 \pm 1.57)$, and Ecklonia radiata $(30.86 \pm 1.48)$.

Densities of total animals and amphipods showed a strong inverse exponential relationship with mean algal thallus width (Fig. 4). Molluscs displayed the same pattern less strongly, while isopod densities were unrelated to mean algal thallus width.

Amphipods were numerically dominant on finely structured algal species, whereas isopods became more dominant on coarsely structured algae (Fig. 5). Molluscs comprised a consistently low proportion of the total epifauna on all algal species.

When isopods were classified by body shape (as 'flat' or 'tubular'), there was a trend for the proportion of flat isopods to increase with increasing algal thallus width (Fig. 6). On Ecklonia radiata, the alga with the widest laminae, flat isopods comprised virtually $100 \%$ of the common isopod taxa.

The finely structured typical/fronded Carpophyllum plumosum var. capillifolium (mean thallus width = $1.55 \mathrm{~mm}, \mathrm{SE}=0.17$ ) supported far more animals than

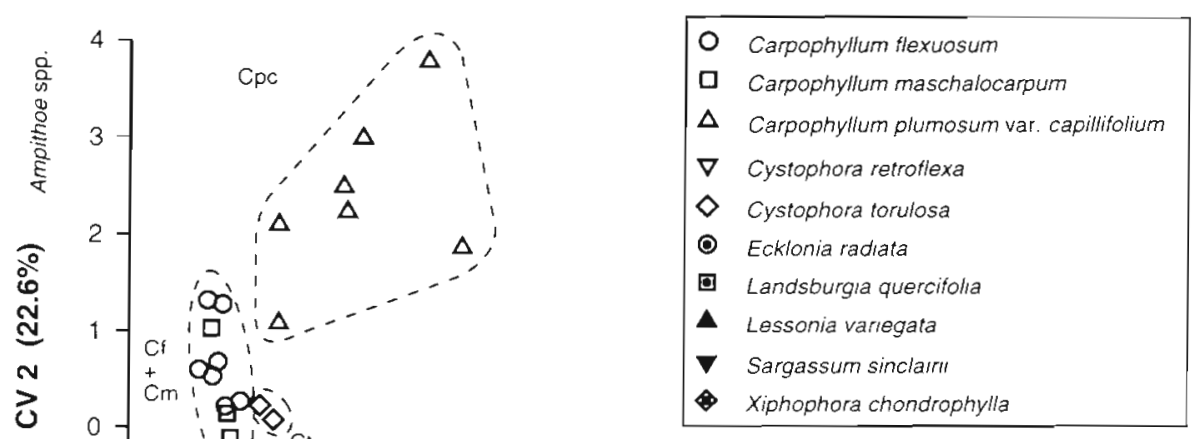

Fig. 2. Canonical discriminant analysis (CDA) plot of the first 2 canonical variates (CVs) summarising trends in densities of the 25 commonest epifaunal taxa on 10 subtidal brown algal species. Epifaunal taxa labelled on axes are those that were identified by the CDA as showing largest density differences among the seaweed species. Each point represents the mean canonical variate score of 5 plants from a particular algal species/site combination. Percentages associated with each $\mathrm{CV}$ refer to the proportion of total variation accounted for by that $\mathrm{CV}$ 


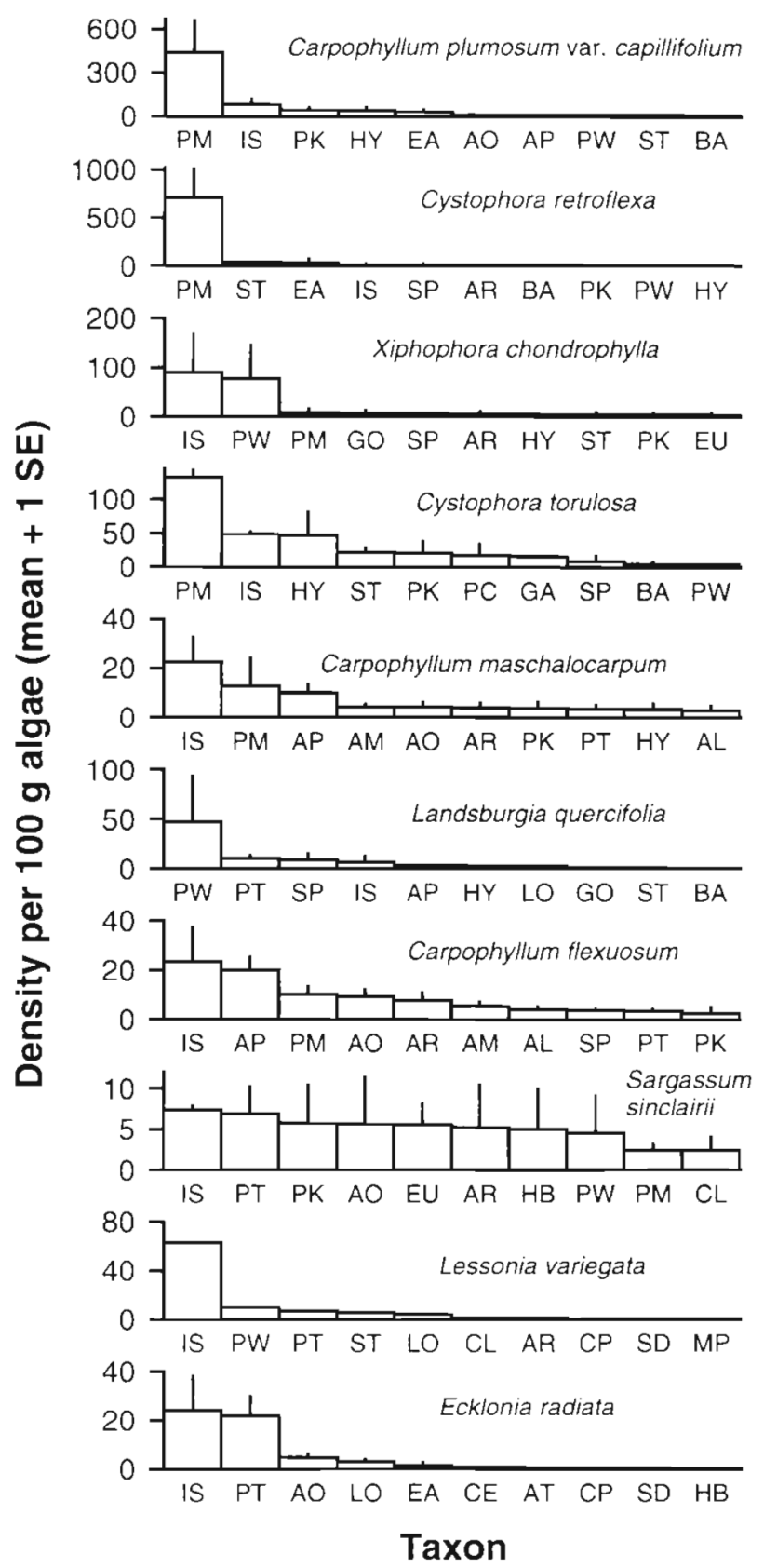

Fig. 3. Rank abundances of epifauna on seaweeds for all sites sampled. Bars represent $1 \mathrm{SE}$. Absence of error bars on Lessonia variegata graph is because only 1 site was sampled. Algal species are ranked in ascending order of mean thallus width. $\mathrm{AL}=$ Ampithoe lessoniae, $\mathrm{AM}=$ Amphoroidea media, $\mathrm{AO}=$ Aora maculata, $\mathrm{AP}=$ Ampithoe spp., $\mathrm{AR}=$ Arcturidae, $\mathrm{AT}=$ Atyloella spp., $\mathrm{BA}=$ Batedotea elongata $\mathrm{CE}=$ Ceinidae, $\mathrm{CP}=$ Cantharidus purpureus, $\mathrm{EA}=$ Eatoniella spp., $\mathrm{GA}=$ Gammaropsis spp., $\mathrm{GO}=$ Gondogeneia spp., $\mathrm{HB}=$ Hippolyte bifidirostris, $\mathrm{HY}=$ Hyale spp., IS = Ischyroceridae, $\mathrm{LO}=$ Amphoroidea longipes, $\mathrm{MP}=$ Maurea punctulata, $\mathrm{PC}=$ Parapherusa crassipes, $\mathrm{PK}=$ Podocerus karu, $\mathrm{PM}=$ Podocerus manawatu, $\mathrm{PT}=$ Plakarthrium typicum, $\mathrm{PW}=$ Podocerus wanganui, SD = Siriella denticulata, SP = miscellaneous Sphaeromatidae, ST = Stenothoe spp. the coarsely structured $C$. plumosum $(2.85 \pm 0.19)$ at Waterfall Crest where the 2 growth forms co-occurred (Table 3). Although the mean densities were not strikingly different, 2 of the 5 finely structured plants held over 1000 animals per $100 \mathrm{~g}$, whereas the highest density on the coarse plants was only 240 animals per $100 \mathrm{~g}$. At Okakari Point we sampled adjacent beds ( $3 \mathrm{~m}$ apart) of finely dissected typical/fronded $C$. plumosum var. capillifolium and frondless/epiphytized
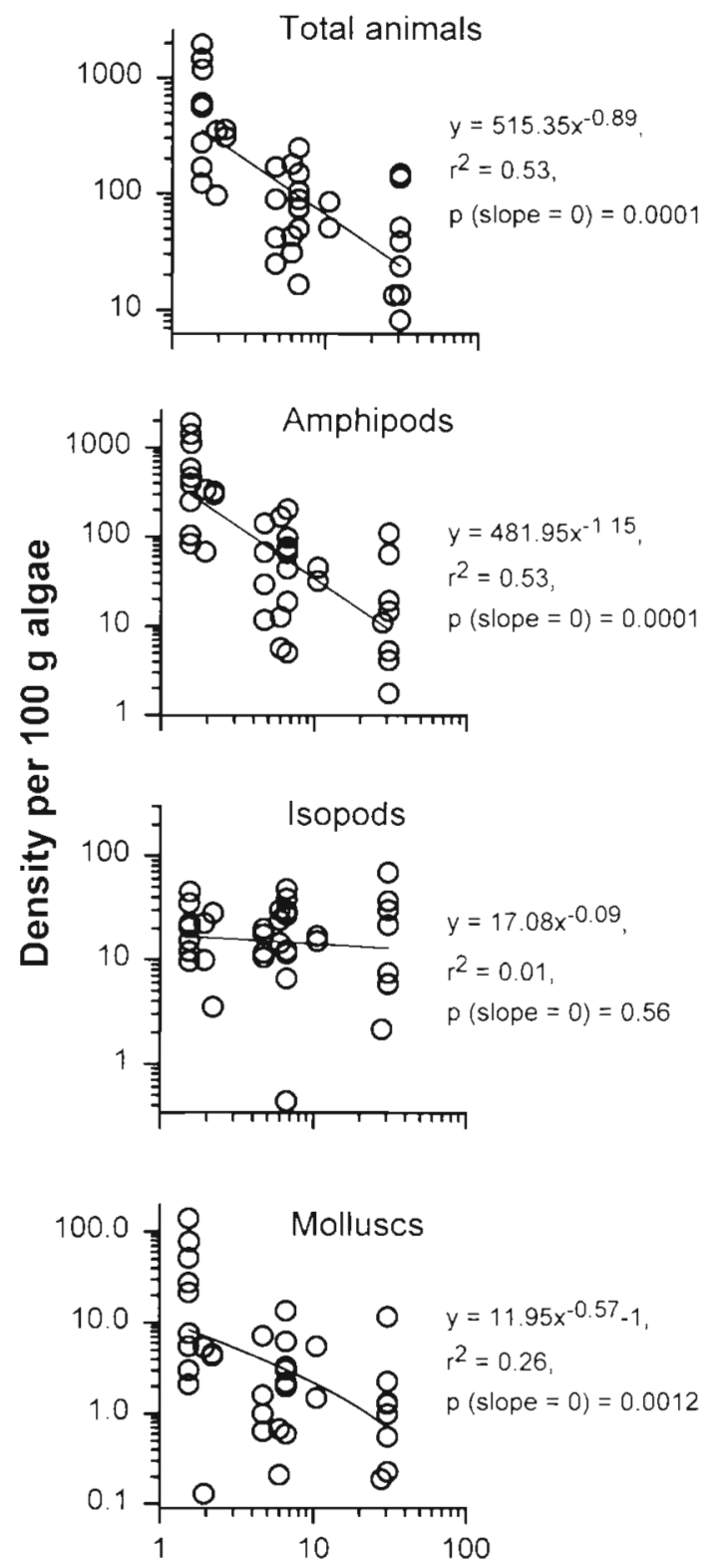

\section{Algal thallus width $(\mathrm{mm})$}

Fig. 4. Epifaunal densities versus mean algal thallus width Each point represents the mean density of epifauna from 5 plants from a particular algal species/site combination 


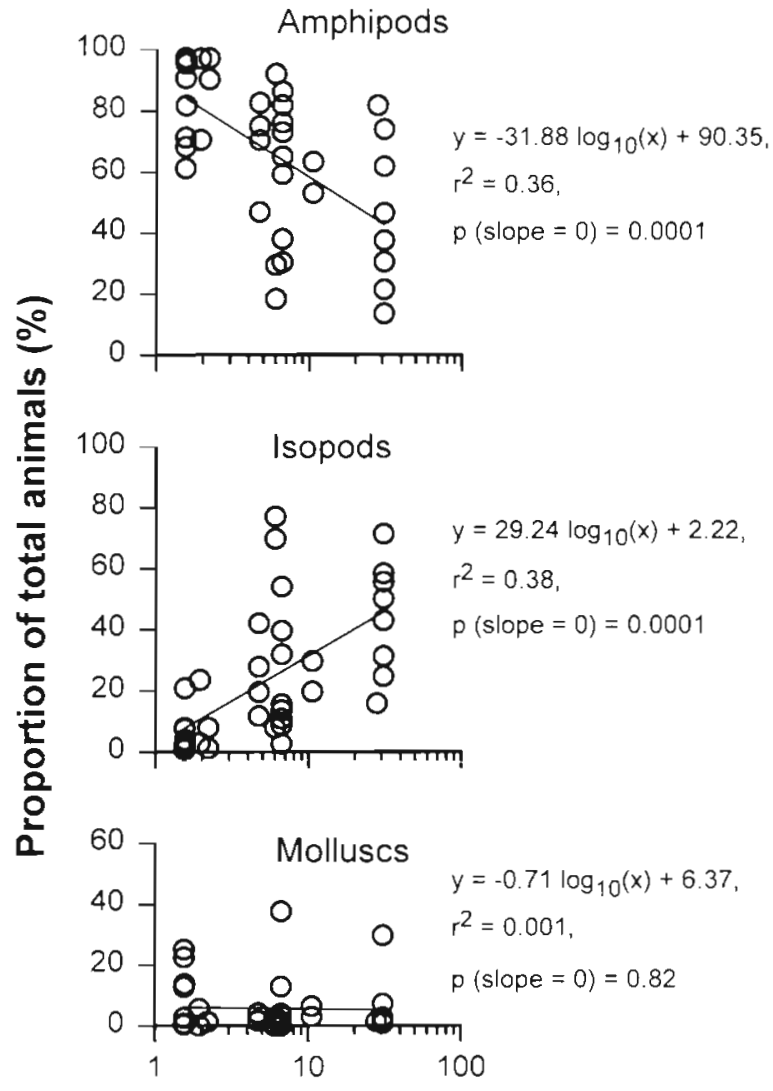

Algal thallus width ( $\mathrm{mm}$ )

Fig. 5. Proportion of total animals versus mean algal thallus width. Each point represents the mean from 5 plants from a particular algal species/site combination

C. plumosum var. capillifolium (mean thallus width $=$ $3.34 \mathrm{~mm}, \mathrm{SE}=0.25)$. Again, the finely structured typical/fronded form supported far more animals than the coarser frondless/epiphytized form (Table 3 ). In the reciprocal transplant experiment, epifauna recolonized denuded typical/fronded plants at far higher densities in beds of frondless/epiphytized individuals than vice versa, indicating that differences in epifaunal densities between the growth forms were due to differences between the plants, not the sites they were growing in (Table 4).

Table 3. Mean densities ( $\pm 1 \mathrm{SE}$ ) of epifauna per $100 \mathrm{~g}$ algae $(\mathrm{n}=5$ plants $)$ on different growth forms of Carpophyllum plumosum

\begin{tabular}{|lccc|}
\hline Site & $\begin{array}{c}\text { Typical/fronded } \\
\text { C. plumosum } \\
\text { var. capillfolium }\end{array}$ & C. plumosum & $\begin{array}{c}\text { Frondless/epiphytized } \\
\text { C. plumosum } \\
\text { var. capillifolium }\end{array}$ \\
\hline $\begin{array}{l}\text { Waterfall Crest } \\
\text { Okakari Point }\end{array}$ & $\begin{array}{r}587 \pm 234 \\
1460 \pm 238\end{array}$ & $149 \pm 39$ & $104 \pm 28$ \\
\hline
\end{tabular}

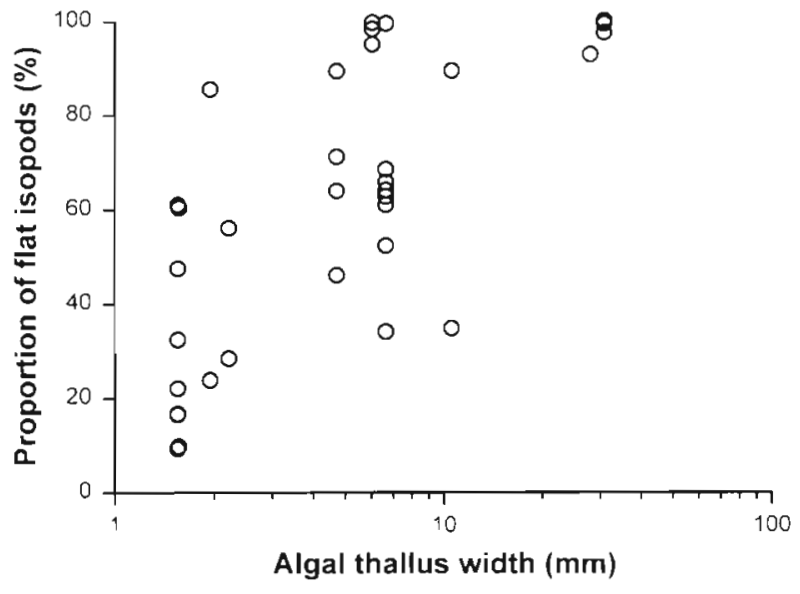

Fig. 6. Proportion of summed flat and tubular isopods comprised of flat isopods versus mean algal thallus width. Each point represents the mean from 5 plants from a particular algal species/site combination

\section{Depth-related variation of epifauna on Ecklonia radiata}

Total epifaunal densities on Ecklonia radiata peaked at $6 \mathrm{~m}$ depth at both sites sampled (Fig. 7). Densities of the 3 most common taxa then steadily declined to almost zero at the reef base at Splendid Reef $(18 \mathrm{~m})$, but showed a slight increase at the bottom of the reef at Ray Rock $(15 \mathrm{~m})$. At both sites Plakarthrium typicum and Ischyroceridae reached maximal densities at $6 \mathrm{~m}$, while Amphoroided longipes declined steadily with increasing depth from $3 \mathrm{~m}$. The ranking of densities of the 3 taxa remained fairly constant over the depth range $(P$. typicum $>$ Ischyroceridae $>A$. longipes $)$.

\section{DISCUSSION}

This study is the first to describe basic patterns in the distribution and abundance of large mobile epifauna on subtidal New Zealand seaweeds. The $1 \mathrm{~mm}$ mesh we used retained many amphipods, isopods and gastropods, but not harpacticoid copepods, nematodes, and most juvenile crustaceans. The epifauna found in our study were taxonomically similar to those found elsewhere in the world, although quantitative comparisons between studies are difficult to make because of differences in mesh sizes used to capture the animals. Very briefly, studies in Tasmania (Edgar 1983a), South Africa (Allen \& Griffiths 1981), California (USA) (Coyer 1984), Japan 
Table 4. Mean densities ( $\pm 1 \mathrm{SE}$ ) of epifauna per $100 \mathrm{~g}$ algae ( $\mathrm{n}=5$ plants) on typical/fronded versus frondless/epiphytized Carpophyllum plumosum var. capillifolium in naturally occurring adjacent beds, and on denuded plants $10 \mathrm{~d}$ after trans. plantation into reciprocal beds

\begin{tabular}{|lcc|}
\hline & $\begin{array}{l}\text { Naturally } \\
\text { occurring }\end{array}$ & $\begin{array}{c}\text { After transplantation } \\
\text { into reciprocal bed }\end{array}$ \\
\hline Typical/fronded & $716 \pm 152$ & $357 \pm 137$ \\
Frondless/epiphytized & $46 \pm 20$ & $56 \pm 17$ \\
\hline
\end{tabular}

(Mukai 1971), and Denmark (Hagerman 1966) have variously found amphipods, isopods, tanaids, mysid and caridean shrimps, gastropods, and polychaetes to be dominant on brown algae, with harpacticoid copepods, ostracods, nematodes, mites and bivalve spat often found in very high numbers when mesh sizes down to $0.1 \mathrm{~mm}$ were used.

It is unclear whether the patterns we described for epifauna sampled in winter and early spring would persist throughout the year. Edgar (1983b) found that peak abundances of almost all epifauna on the Tasmanian algae that he investigated occurred in late summer or early autumn. However, in New Zealand, monthly sampling over a year of epifauna on Carpophyllum flexuosum and Ecklonia radiata at Matatuahu Point (see Fig, 1) by Taylor (1991) revealed taxa peaking in roughly even numbers across all 4 seasons. Hicks (1977b) likewise found that harpacticoid copepod density maxima occurred throughout the year on several species of algae at Wellington.

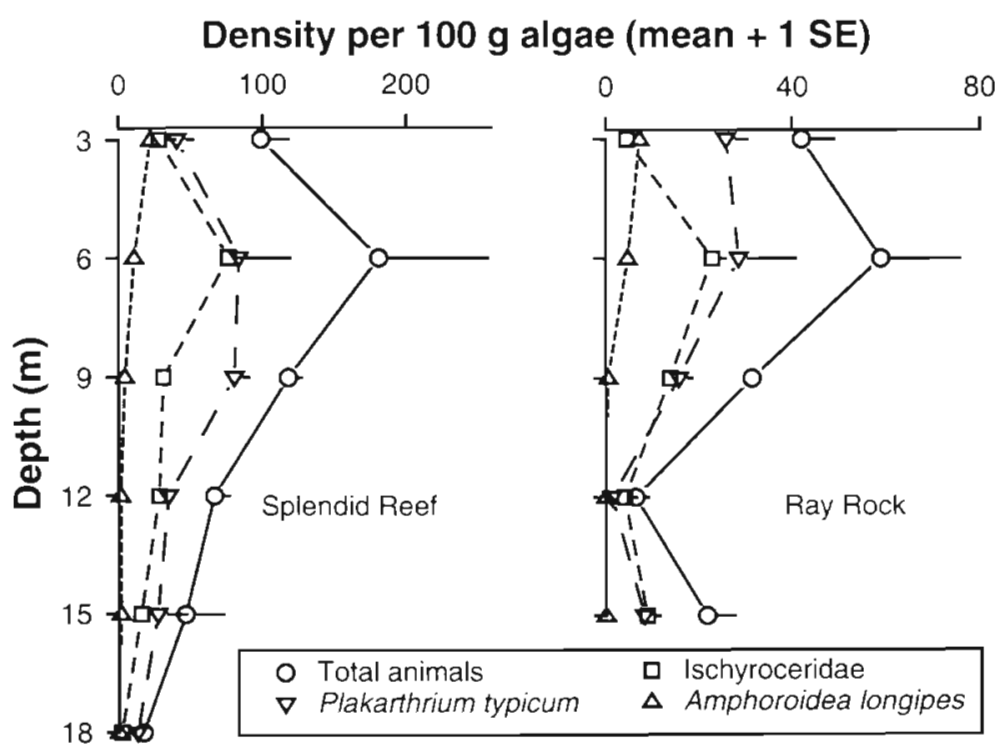

Fig. 7. Depth profile of epifaunal abundances on Ecklonia radiata at 2 sites ( $\mathrm{n}=3$ plants)

\section{Effects of algal morphology}

Differences in the morphology of the plants apparently accounted for much of the variation in epifaunal densities among the algal species investigated, with finely structured algal species tending to support higher epifaunal densities than coarsely structured ones. The differences in epifaunal densities between finely and coarsely structured growth forms of Carpophyllum plumosum suggest that plant morphology rather than some internal property determines the densities of many epifaunal taxa (with the caveat that internal factors could still have been important if they covaried with morphology). There are several ways in which plant morphology may affect epifauna.

(1) Finely structured plants may provide a greater surface area per wet weight for periphyton and other food items. Most epifaunal taxa on some algal species readily colonise artificial habitats once these have been covered by diatoms and algal epiphytes, suggesting that these are important food sources for epifauna on natural plants (Russo 1988, Edgar 1991a, b).

(2) Finely structured plants may provide better refuge from predation. Numerous studies in varied aquatic environments have shown that vegetation shelters associated animals from predation to at least some extent (see reviews by Coull \& Wells 1983, Gotceitas \& Colgan 1989), and finely structured plants usually provide better shelter than do coarsely structured plants (but see Holmlund et al. 1990). Epifaunal crustaceans are preyed upon by reef fish of many species in New Zealand (Jones 1988, Taylor 1991) and elsewhere (e.g. Schmitt \& Holbrook 1984).

(3) Where the epifauna are using the plant primarily as an attachment point from which to resist being dislodged by water movement while they filter feed (e.g. Edgar 1983a), finely structured plants may provide a more suitable surface for grasping. Amphipods from exposed coasts have prehensile pereopods (walking legs) used to tightly grip their substratum, which must be a cylindrical object of a diameter small enough to be encircled by the dactyl and propodus (Caine 1978, Vader 1983, Aoki \& Kikuchi 1990).

Experiments are needed to determine whether the trend for dorso-ventrally flattened isopods rather than tubular ones to predominate on wide-bladed algae is due to active selection of algae by the isopods. Fish predation rates may be higher on isopods which do not match their host alga's morphology. Shrimps living on pelagic Sargassum have body shapes and colouration 
matching the parts of the host plant on which they live, which presumably helps to camouflage them from fish predators (Hacker \& Madin 1991). Alternatively, the structure of the isopods' bodies may determine in a more direct manner which algae they can physically hold on to. Dorso-ventrally flattened isopods may move onto wider-bladed algae as they grow - it is possible that the individuals found on finely structured plants were small, or were living on the stipes rather than on the fronds.

\section{Degree of host-plant specificity}

In our study, most of the 10 algal species investigated had epifaunas which were distinct in multivariate space, but the individual epifaunal taxa (including many of those identified to species level) generally occurred on more than 1 algal species. A similar lack of host-plant specificity has been recorded for Tasmanian (Edgar 1983a) and Hawaiian (Russo 1990) algal epifaunas. This may be explained by reference to their feeding habits. The extent to which epifauna graze the tissue of their host plant is contentious (Bell 1991, Duffy \& Hay 1991), but most invertebrate grazers of marine algae investigated to date have been found to be generalists (Hay et al. 1990 and references therein). Epifauna feeding on items other than the host plant will also tend not to show specificity, as their prey items (periphyton, algal epiphytes, other epifauna, plankton, etc.) are probably not host-plant specific themselves [e.g. Russo (1988) and Edgar (1991a, b) have shown that periphyton readily colonizes artificial substrata].

Many epifaunal taxa, especially amphipods and isopods, are demersal zooplankters, and spend time in the water column at night, resettling on various substrata before daybreak (e.g. Hobson \& Chess 1976). Depending on how far they have moved, individuals resettling may have a limited choice of algal species and simply settle on whatever is available, or alternatively, may be non-selective if they do have a choice, thus displaying low host specificity either way. The ischyrocerid amphipods probably do this, being abundant on all algal species, very active in the water col- umn at night, and apparently not discriminating among settlement substrata to a great extent. At Goat Island they recolonize experimentally denuded seaweed plants within days (White 1989), and have also been trapped in large numbers entering the water column at night from sand and coralline turf (Moltschaniwskyj 1989).

\section{Depth-related variation of epifauna on Ecklonia radiata}

Ecklonia radiata was the only alga at Leigh found continuously from the intertidal to the bottom of the reef at about $18 \mathrm{~m}$. Epifaunal densities along this depth profile increased from a depth of $3 \mathrm{~m}$ to a peak at $6 \mathrm{~m}$, and then declined with increasing depth. Gallahar \& Kingsford (1993) found that abundances of mobile epifauna on E. radiata varied much less predictably with depth at 2 sites near Sydney. In fact, densities of many common taxa were highest on the deepest plants they sampled $(14 \mathrm{~m})$. Declines in epifaunal abundance with increasing depth have been tentatively attributed to decreased water movement, which lessens opportunities for filter feeding (Fenwick 1976, Edgar 1983a) or causes increased sedimentation which may somehow negatively affect epifauna (Hagerman 1966). Edgar (1991b) suggests that the limiting microalgal food base could be reduced due to diminished light levels. We have no explanation for the low epifaunal densities found at $3 \mathrm{~m}$ depth.

\section{Ecological significance}

Seaweed epifaunal abundances can be very high per unit area of reef, especially in dense beds of finely dissected seaweed species such as Carpophyllum plumosum var. capillifolium (Table 5). Here the epifauna provide a potentially important trophic link between fish and primary producers such as the host seaweed with its associated periphyton, and phytoplankton from the surrounding seawater (in cases where the epifauna are filter feeding). In northeastern New Zealand, epifaunal

Table 5. Densities of total epifauna $\mathrm{m}^{-2}$ of substratum for 3 common bed-torming seaweed species. Plant density data from R. Taylor \& R. Cole (unpubl, datal

\begin{tabular}{|c|c|c|c|}
\hline Algal species & Animals per plant & $\begin{array}{l}\text { Plants } \mathrm{m}^{-2} \text { of } \\
\text { substratum }\end{array}$ & $\begin{array}{c}\text { Animals } \mathrm{m}^{-2} \text { of } \\
\text { substratum }\end{array}$ \\
\hline Carpophyllum plumosum var. capillifolium & 585 & 277 & 162045 \\
\hline Carpophyllum flexuosum & 147 & 25 & 3675 \\
\hline Ecklonia radiata & 150 & 11 & 1650 \\
\hline
\end{tabular}


crustaceans are major dietary items for juvenile reef fish (Jones 1988), and epifaunal gastropods occur in the diet of many adult reef fishes (Russell 1983). Most fish in New Zealand and other temperate waters are carnivorous, with few species feeding directly on macroalgae (Choat 1982, Russell 1983, Horn 1989).

The effects of such large numbers of epifauna on their host seaweeds will remain largely unknown in the absence of long-term controlled epifaunal removal experiments, which are technically difficult to conduct given the ability of the epifauna to rapidly recolonize denuded plants.

Acknowledgements. We are grateful to H. Clark, D. Cowley, B. Creese, G. Hicks, J. Miller, M. Miller, W. Nelson, C. Paulin, G. Poore, and B. Stephenson for taxonomic advice. R. Gorter kindly collected Lessonia for us. We thank R. Babcock, B. Ballantine, J. Evans, B. Foster, C. Jacoby, B. Robbins, C. Syms, C. Trowbridge, and the anonymous referees for their comments on the manuscript. We also thank the New Zealand Department of Conservation for permitting us to sample within the Cape Rodney to Okakari Point Marine Reserve.

\section{LITERATURE CITED}

Allen, J. C., Griffiths, C. L. (1981). The fauna and flora of a kelp bed canopy. S. Afr. J. Zool. 16: 80-84

Aoki, M., Kikuchi, T (1990). Habitat adaptations of caprellid amphipods and the importance of epiphytic secondary habitats in a Sargassum patens bed in Amakusa, southern Japan. Publ. Amakusa Mar Biol. Lab. Kyushu Univ. 10: $123-133$

Barnard, J. L. (1972). The marine fauna of New Zealand: algae-living littoral Gammaridea (Crustacea Amphipoda). N.Z. Oceanogr. Inst. Mem. 62: 1-216

Barnard, J. L., Karaman, G. S. (1991). The families and genera of marine gammaridean Amphipoda (except marine gammaroids). Rec. Aust. Mus. (Suppl.) 13: 1-866

Bell, S. S. (1991). Amphipods as insect equivalents? An alternative view. Ecology 72: 350-354

Brawley, S. H., Fei, X. G. (1987). Studies of mesoherbivory in aquaria and in an unbarricaded mariculture farm on the Chinese coast. J. Phycol. 23: 614-623

Bray, R. N., Ebeling, A. W. (1975). Food, activity, and habitat of three 'picker-type' microcarnivorous fishes in the kelp forests off Santa Barbara, California. Fish. Bull. U.S. 73: $815-829$

Caine, E. A. (1977). Feeding mechanisms and possible resource partitioning of the Caprellidae (Crustacea: Amphipoda) from Puget Sound, USA. Mar. Biol. 42: $331-336$

Caine, E. A. (1978). Habitat adaptations of North American caprellid Amphipoda (Crustacea). Biol. Bull. 155: 288-296

Choat, J. H. (1982). Fish feeding and the structure of benthic communities in temperate waters. A. Rev. Ecol. Syst. 13: $423-449$

Choat, J. H., Andrew, N. L. (1986). Interactions amongst species in a guild of subtidal benthic herbivores. Oecologia 68: $387-394$

Choat, J. H., Schiel, D. R. (1982). Patterns of distribution and abundance of large brown algae and invertebrate herbivores in subtidal regions of northern New Zealand. J. exp. mar. Biol. Ecol. 60: 129-162
Coull, B. C., Wells, J. B. J. (1983). Refuges from fish predation: experiments with phytal meiofauna from the New Zealand rocky intertidal. Ecology 64: 1599-1609

Coyer, J. A. (1984). The invertebrate assemblage associated with the giant kelp, Macrocystis pyrifera, at Santa Catalina Island, California: a general description with emphasis on amphipods, copepods, mysids, and shrimps Fish. Bull, U.S. 82: 55-66

Duffy, J. E. (1990). Amphipods on seaweeds: partners or pests? Oecologia 83: 267-276

Duffy, J. E., Hay, M. E. (1991). Amphipods are not all created equal: a reply to Bell. Ecology 72: $354-358$

Edgar, G. J. (1983a). The ecology of south-east Tasmanian phytal animal communities. I. Spatial organisation on a local scale. J. exp. mar. Biol. Ecol. 70: 129-157

Edgar, G. J. (1983b). The ecology of south-east Tasmanian phytal animal communities. II. Seasonal change in plant and animal populations. J. exp. mar. Biol. Ecol. 70 : $159-179$

Edgar, G. J. (1991a). Artificial algae as habitats for mobile epifauna: factors affecting colonization in a Japanese Sargassum bed. Hydrobiologia 226: 111-118

Edgar, G. J. (1991b). Distribution patterns of mobile epifauna associated with rope fibre habitats within the Bathurst Harbour estuary, south-western Tasmania. Estuar coast. Shelf Sci. 33: 589-604

Edgar, G. J., Moore, P. G. (1986). Macro-algae as habitats for motile macrofauna. Monogr. Biol. 4: 255-277

Evans, J. H. (1992). Leigh climate report 1991. Leigh Lab. Bull 27: 1-56

Fenwick, G. D. (1976). The effect of wave exposure on the amphipod fauna of the alga Caulerpa brownii. J. exp. mar. Biol. Ecol. 25: 1-18

Gallahar, N. K., Kingsford, M. J. (1993). The influence of depth on assemblages of small mobile invertebrates on the kelp, Ecklonia radiata. In: Battershill, C. N., Schiel, D. R., Jones, G. P., Creese, R. G., MacDiarmid, A. B. (eds.) Proceedings of the Second International Temperate Reef Symposium, 7-10 January 1992, Auckland, New Zealand. NIWA Marine, Wellington, p. 163-168

Gotceitas, V., Colgan, P. (1989). Predator foraging success and habitat complexity: quantitative test of the threshold hypothesis. Oecologia 80: 158-166

Grace, R. V. (1983). Zonation of sublittoral racky bottom. marine life and its changes from the outer to the inner Hauraki Gulf, north-eastern New Zealand. Tane 29: $97-108$

Hacker, S. D., Madin, L. P. (1991). Why habitat architecture and color are important to shrimps living in pelagic Sargassum: use of camouflage and plant-part mimicry. Mar. Ecol. Prog. Ser. 70: 143-155

Hacker, S. D., Steneck, R. S. (1990). Habitat architecture and the abundance and body-size-dependent habitat selection of a phytal amphipod. Ecology 71. 2269-2285

Hagerman, L. (1966). The macro- and microfauna associated with Fucus serratus L., with some ecological remarks. Ophelia 3: 1-43

Hardy, G. S. (1989). The genus Forsterygion Whitley and Phillips, 1939 (Pisces: Tripterygiidae) in New Zealand and Australia, with descriptions of two new species. J. nat. Hist. 23: 491-512

Hay, M. E., Duffy, J. E., Fenical, W. (1990). Host-plant specialization decreases predation on a marine amphipod: an herbivore in plant's clothing. Ecology 71: 733-743

Hicks, G. R. F. (1977a). Species composition and zoogeography of marine phytal harpacticoid copepods from Cook Strait, and their contribution to total phytal meiofauna. 
N.Z. J. mar. Freshwat. Res. 11:441-469

Hicks, G. R F. (1977b). Species associations and seasonal population densities of marine phytal harpacticoid copepods from Cook Strait. N.Z. J. mar. Freshwat. Res. 11: $621-643$

Hobson, E. S. Chess, J. R. (1976). Trophic interactions among fishes and zooplankters near shore at Santa Catalina Island, California. Fish. Bull. U.S. 74:567-598

Holmlund, M. B., Peterson, C. H., Hay, M. E. (1990). Does algal morphology affect amphipod susceptibility to fish predation? J. exp. mar. Biol. Ecol. 139: 65-83

Horn, M. H. (1989). Biology of marine herbivorous fishes. Oceanogr. mar. Biol. A. Rev. 27: 167-272

Hurley, D. E. (1961). A checklist and key to the Crustacea Isopoda of New Zealand and the Subantarctic Islands. Trans. R. Soc. N.Z. (Zool.) 1(20): 259-292

Hurley, D. E., Jansen, K. P. (1977). The marine fauna of New Zealand: Family Sphaeromatidae (Crustacea Isopoda: Flabellifera). N.Z. Oceanogr. Inst. Mem. 63: 1-95

Jansen, K. P. (1971). Ecological studies on intertidal New Zealand Sphaeromatidae (Isopoda: Flabellifera). Mar. Biol. 11: 262-285

Jones, G. P. (1988). Ecology of rocky reef fish of north-eastern New Zealand: a review. N.Z. J. mar. Freshwat. Res. 22: $445-462$

Kingsford, M. J., Choat, J. H. (1985). The fauna associated with drift algae captured with a plankton-mesh purse seine net. Limnol. Oceanogr. 30: 618-630

Lindauer, V. W., Chapman, V. J., Aiken, M. (1961). The marine algae of New Zealand. II: Phaeophyceae. Nova Hedwigia 3: 129-350

Lowry, J. K. (1981). The amphipod genus Cerapus in New Zealand and subantarctic waters (Corophioidea, Ischyroceridae). J. nat. Hist. 15: 183-211

McCain, J. C. (1969). New Zealand Caprellidae (Crustacea: Amphipoda). N.Z. J. mar. Freshwat. Res. 3: 286-295

McLay, C. L. (1988). Brachyura and crab-like Anomura of New Zealand. Leigh Lab. Bull. 22: 1-463

Melrose, M. J. (1975). The marine fauna of New Zealand: Family Hymenosomatidae (Crustacea, Decapoda, Brachyura). N.Z. Oceanogr. Inst. Mem. 34: 1-123

Moltschaniwskyj, N. A. (1989). The settlement and recruitment of Upeneichthys lineatus (Pisces: Mullidae). M.Sc. thesis, University of Auckland

Morton, J. E., Miller, M. C. (1968). The New Zealand sea shore. Collins, London

Mukai, H. (1971). The phytal animals on the thalli of Sargassum serratifolium in the Sargassum region, with reference to their seasonal fluctuations. Mar. Biol. 8: 170-182

Nelson, W. G. (1979). An analysis of structural pattern in an eelgrass (Zostera marina L.) amphipod community. J. exp. mar. Biol. Ecol 39: 231-264

Paulin, C., Stewart, A., Roberts, C., McMillan, P. (1989). New Zealand fish: a complete guide. Natl Mus. N.Z. misc. Ser. 19: $1-279$

This article was submitted to the editor
Poore, G. C. B. (1981). Marine Isopoda of the Snares Islands New Zealand - 1. Gnathiidea, Valvifera, Anthuridea, and Flabellifera. N.Z. J. Zool 8: 331-348

Poore, G. C. B., Lew Ton, H. M. (1993). Idoteidae of Australia and New Zealand (Crustacea: Isopoda: Valvifera). Invertebr. Taxon. 7: 197-278

Powell, A. W. B. (1979). New Zealand Mollusca: marine, land and freshwater shells. Collins, Auckland

Richardson, L. R., Yaldwyn, J. C. (1958). A guide to the natant decapod Crustacea (shrimps and prawns) of New Zealand. Tuatara $7: 17-41$

Riek, E. F. (1976). The marine caddisfly family Chathamiidae (Trichoptera). J. Aust. Entomol. Soc. 15: 405-419

Roland, W. (1978). Feeding behaviour of the kelp clingfish Rimicola muscarum residing on the kelp Macrocystis integrifolia. Can. J. Zool. 56: 711-712

Russell, B. C. (1983). The food and feeding habits of rocky reef fish of north-eastern New Zealand. N.Z. J. mar. Freshwat. Res. 17: 121-145

Russo, A. R. (1988). Detritus and epibiota on artificial substrata: the possible role of food in structuring Hawaiian epiphytal amphipod communities. Int. Rev. ges. Hydrobiol. 73: 319-325

Russo, A. R. (1990). The role of seaweed complexity in structuring Hawaiian epiphytal amphipod communities. Hydrobiologia 194: 1-12

SAS Institute Inc. (1987). SAS/STAT ${ }^{\mathrm{TM}}$ guide for personal computers, Version 6 edition. SAS Institute, Inc, Cary, NC

Schiel, D. R. (1988). Algal interactions on shallow subtidal reefs in northern New Zealand: a review. N.Z. J. mar. Freshwat. Res. 22: 481-489

Schmitt, R. J., Holbrook, S. J. (1984). Gape-limitation, foraging tactics and prey size selectivity of two microcarnivorous species of fish Oecologia 63: 6-12

Stoner, A. W. (1980). Abundance, reproductive seasonality and habitat preferences of amphipod crustaceans in seagrass meadows of Apalachee Bay, Florida. Contrib. mar. Sci. 23: 63-77

Taylor, R. B. (1991). Effects of Notolabrus celidotus (Labridae) predation on motile macroalgal epifauna. M.Sc. thesis. University of Auckland

Tegner, M. J., Dayton, P. K. (1987). El Nin̄o effects on southern California kelp forest communities. Adv. ecol. Res. 17: 243-279

Vader, W. (1983). Prehensile pereopods in gammaridean Amphipoda. Sarsia 68: 139-148

White, S. E. (1989). Ecology and behaviour of mobile epifauna on rocky reef macroalgae. M.Sc. thesis, University of Auckland

Wilson, G. D., Thistle, D., Hessler, R. R. (1976). The Plakarthriidae (Isopoda: Flabellifera): déjà vu. Zool. J. Linn. Soc. 58: $331-343$

Zimmerman, R., Gibson, R., Harrington, J. (1979). Herbivory and detritivory among gammaridean amphipods from a Florida seagrass community. Mar. Biol. 54: 41-47

Manuscript first received: March 15, 1994

Revised version accepted: September 20, 1994 